"Förderprogramme spiegeln insofern auch die gesellschaftlichen Bedingungen wider« (Gedächtniszitat nach LFI-4). Gleichzeitig wurden $\mathrm{CO} 2$-Einsparungen und Energieeffizienz bereits seit den 1990er Jahren gefordert, während die Barrierefreiheit erst deutlich später ins Bewusstsein rückte. In diesem Sinne wirkt etwa das Sanitärgewerbe als Multiplikator, da es aufgrund seiner eigenen wirtschaftlichen Interessen über die Programme der Förderbanken informiert (LFI-13).

Dieses UnterKapitel sollte zu einem tieferen Verständnis der Förderstruktur zum barrierefreien bzw. -reduzierten Wohnraum beitragen. Neben der KfW und den Ländern fördern zum Teil auch einzelne Kommunen über kommunale Wohnraumförderprogramme die Akteure ihrer lokalen Wohnungsmärkte. Dieser Aspekt wurde hier nicht behandelt, findet aber - sofern relevant - in den Fallstudien zu Hessen und SachsenAnhalt Einzug (vgl. vor allem Kapitel 7.2.1).

\title{
6.3 Informationell: Wohnberatung zur Wohnungsanpassung
}

Nachdem die bauordnungsrechtlichen Regelungen zum barrierefreien Bauen als verbindliches regulatives Instrumentarium und die Landesförderprogramme als freiewillige ökonomische Steuerung analysiert wurden, richtet sich der Blick im Folgenden auf die Wohnberatung zur Wohnungsanpassung (teils auch »Wohnraumanpassung genannt). Diese Form von informationeller und damit »weicher« Steuerung vervollständigt den hier untersuchten Policy-Mix zum barrierefreien bzw. -reduzierten Wohnraum. Sie fokussiert insbesondere Umbaumaßnahmen im Bestand. Die handlungsleitende Frage für dieses UnterKapitel lautet: Inwiefern fördern die Bundesländer den Aufbau und die Unterhaltung von Wohnberatungsstellen zur Wohnungsanpassung?

Anders als das Bauordnungsrecht und die soziale Wohnraumförderung hat die Wohnberatung in der föderalen Zuständigkeit zwischen Bund, Ländern und Kommunen keinen festen Platz. Einerseits wird sie als Bestandteil der Pflegeberatung verstanden, womit sie eine Pflichtaufgabe der Pflegeversicherungen wäre. Andererseits kann sie zum Leistungsspektrum der Altenhilfe und damit zur freiwilligen kommunalen Daseinsvorsorge gerechnet werden (IP 1: 79ff). Das durch diese unklaren Zuständigkeiten entstehende Vakuum wird von einigen Bundesländern erkannt und mit landespolitischen Maßnahmen gefüllt.

Um die Rolle der Bundesländer in diesem Feld zu erfassen, muss zunächst ein Verständnis dafür entwickelt werden, wie sich die Wohnberatung zur Wohnungsanpassung insgesamt strukturiert. Dafür skizziert Kapitel 6.3.1 die grundlegende Idee dieses Beratungsangebots und Kapitel 6.3.2 führt in die Bundesarbeitsgemeinschaft (BAG) Wohnungsanpassung e.V. ein. Ähnlich wie schon mit der Musterbauordnung und mit dem KfW-Programm "Altersgerecht Umbauen « wird damit ein bundesweiter Referenzrahmen gesetzt. Den Schwerpunkt bildet Kapitel 6.3.3 zu den unterschiedlichen Strukturen der Wohnberatung: Welche Akteure bieten diese Beratungsangebote an und wie finanzieren sie sich? Es gilt zu prüfen, ob und wenn ja welche Rolle den einzelnen Bundesländern hierbei zukommt. Abschließend fasst Kapitel 6.3.4 die gewonnenen Erkenntnisse zusammen. 


\subsubsection{Die Idee der Wohnberatung zur Wohnungsanpassung}

Für eine Beschäftigung mit der Wohnberatung zur Wohnungsanpassung ist zunächst einmal der Beratungsgegenstand selbst erklärungsbedürftig. Die Wohnungsanpassung beschreibt »kleinere bis mittelgradige technische bzw. bauliche Maßnahmen, die aufdie individuellen Bedürfnisse der Menschen mit eingeschränkter Alltagskompetenz ausgerichtet sind "(Joo 2018: 73). Es geht also um die bedarfsgerechte und individuelle Anpassung des vorhandenen Wohnraums, die häufig durch eine Verschlechterung des körperlichen oder geistigen Gesundheitszustands notwendig wird. Durch diese Maßnahmen soll weiterhin ein selbstständiges Leben gesichert werden. Zudem sind die Sturzprävention und die Unterstützung von pflegenden Angehörigen bzw. der häuslichen Versorgung sozialpolitische Leitlinien (BMFSFJ 2018: 23; Lehrmacher-Dubberke et al. 2015: 136; Schneider 2013: 158f.).

Die Wohnberatung als Beratungsleistung zur Wohnungsanpassung prüft einerseits die baulichen und technischen Möglichkeiten in Abhängigkeit von den individuellen Bedürfnissen der Betroffenen, ist andererseits aber auch bei der Finanzierung der Umbaumaßnahmen und der dazugehörigen Antragstellung behilflich (Joo 2018: 3; LehrmacherDubberke et al. 2015: 136). Je nach Tätigkeitsfeld unterstützen Wohnberater*innen nicht nur einzelne Mieter"innen oder Selbstnutzer"innen von Wohneigentum, sondern auch Institutionen wie kommunale Einrichtungen oder Wohnungsunternehmen. Genauso können die Vermittlung an entsprechende Handwerksleistungen, die Netzwerkarbeit zu Akteuren der Pflege- und Wohnungsmärkte sowie Öffentlichkeitsarbeit als Aufgaben wahrgenommen werden (Lehrmacher-Dubberke et al. 2015: 136).

Die Zielgruppen der Wohnberatung sind prinzipiell alle Menschen mit einem Bedarf zur Wohnungsanpassung, wobei die Expert*inneninterviews zeigen, dass Hochbetagte über 80 Jahren sowie deren Angehörige den Großteil der Ratsuchenden ausmachen. Vielfach liegt dann bereits eine akute Problemlage durch eine Verschlechterung der gesundheitlichen Situation vor. Dennoch finden auch jüngere Menschen mit Behinderungen den Weg in die Beratungsstellen, zum Beispiel ebenfalls durch eine Veränderung des Gesundheitszustandes oder bedingt dadurch, dass die älter werdenden Angehörigen zusätzliche bauliche Unterstützungen benötigen. Die präventive Beratung war hingegen bislang eher selten, wobei hier ein Wandel stattzufinden scheint. Verstärkt kämen inzwischen auch Menschen kurz vor Renteneintritt, die ohnehin ihr Eigenheim modernisieren. Einerseits habe die öffentliche Thematisierung zum Pflegenotstand und den Herausforderungen in den ambulanten und stationären Systemen zu einer intensiveren Auseinandersetzung mit dem Thema geführt (Wohnberatungsstelle1: 13f.), andererseits hätte die Generation der heute ca. 50- bis 70-Jährigen häufiger als ihre Elterngeneration Erfahrungen darin, Angehörige zu pflegen. Auch dies führt zu einer stärkeren Sensibilisierung (IP 54: 67ff.).

Bei dieser Skizzierung der grundlegenden Idee der Wohnungsanpassung, der dazugehörigen Wohnberatung sowie der primären Nutzergruppe dieser Beratungsleistung ist eine wichtige Einschränkung vorzunehmen: Der Begriff des/der Wohnberater*in ist in Deutschland nicht geschützt, was einen intransparenten Markt mit vielen verschiedenen staatlichen und nichtstaatlichen Anbieter*innen zur Folge hat (Schneider 2013: 
158; Joo 2018: 225ff., 261). Die Bundesarbeitsgemeinschaft Wohnungsanpassung versucht, diesen Markt über Qualitätsstandards und Zertifizierungen zu strukturieren.

\subsubsection{Bundesarbeitsgemeinschaft (BAG) Wohnungsanpassung e.V.}

In den späten 1980er Jahren entstehen die ersten Wohnberatungsstellen, gleichzeitig verschaffen verschiedene Studien und Modellprojekte zu Anpassungsmaßnahmen im Bestand dem Thema Aufwind. Hervorzuheben ist das Bundesprogramm zum Experimentellen Wohnungs- und Städtebau "Ältere Menschen und ihr Wohnquartier« von 1988 bis 1993 (BAG Wohnungsanpassung 2019; Schneider 2013: 156). Seit 1995 finanzieren die Pflegekassen zudem entsprechende Umbaumaßnahmen (BMFSFJ 1999), sodass die Zeit von der Mitte der 1980er bis Mitte der 1990er Jahre als Beginn der Wohnberatungsstellen zur Wohnungsanpassung bezeichnet werden kann. Der eingetragene Verein BAG Wohnungsanpassung wurde 1994 zur Vernetzung und zum Erfahrungsaustausch der bestehenden Beratungsstellen gegründet und stellt damit eine Form gesellschaftlicher Selbstregelung dar (Schneider 2013: 158).

Bis heute hat die BAG Wohnungsanpassung ihr Aufgabenspektrum sukzessive erweitert. Einerseits gehört inzwischen die Interessenvertretung zur Verbesserung der Arbeit der Wohnberater*innen zu einem expliziten Tätigkeitsfeld (BAG Wohnungsanpassung 2019; IP 16: 116ff.; Wohnberatungsstelle-2: 38f.), andererseits wird die Entwicklung von Qualitätsstandards, umgesetzt in zertifizierten Schulungen und berufsbegleitenden Fortbildungen, zunehmend wichtiger (BAG Wohnungsanpassung 2019; Schneider 2013: 159f.). Es handelt sich um eine Qualifizierung mit 130 Unterrichtseinheiten, in der die bereits im Kapitel 6.3.1 erläuterten Tätigkeitsfelder der Wohnberatung vermittelt werden. Ein Blick auf die Zielgruppen dieser Schulungen zeigt dabei die Breite des Feldes auf:

»Alle Interessierten des Themenfeldes 'Wohnen im Alter und bei Behinderung, Mitarbeiter in Pflegestützpunkten, Wohnberatungsstellen, Planungs- und Architekturbüros, Handwerksbetrieben, Wohnungsunternehmen, Ämtern und Behörden, Pflegekassen, Krankenkassen, Berufsgenossenschaften, therapeutischen Praxen, Pflegediensten und Sozialstationen sowie Freiberufliche in der Wohnberatung « (BAC Wohnungsanpassung/Pegasus $\mathrm{CmbH}$ 2020: 2).

Die Qualitätsstandards der BAG werden vielfach auch in die Länder getragen, wobei die BAG-Schulungen inhaltlich und zeitlich meist umfassender als die Grundlagenschulungen in den Ländern - bspw. für ehrenamtliche Wohnberater*innen - sind. Zertifizierte BAG-Schulungen werden in Bayern, Niedersachen und Berlin angeboten (Wohnberatungsstelle-1: 12; IP 16: 65f.; IP 54: 41).

Die BAG ist demnach ein wichtiger Akteur zur Vernetzung der Wohnberatungsstellen untereinander, in der politischen Lobbyarbeit des Berufsfeldes sowie in der Qualitätssicherung des rechtlich nicht geschützten Berufs »Wohnberater"in«. Insgesamt soll aber nicht der Eindruck entstehen, dass alle Akteure, die Wohnberatungen anbieten, in der BAG organisiert wären: Schon die Entrichtung eines jährlichen Mitgliedsbeitrages wäre insbesondere für allgemeinere Beratungsstellen, die lediglich $\mathrm{zu}$ einem kleineren Stellenanteil zusätzlich Wohnberatung anbieten, eine Kostenfrage (IP 14: 17f.; IP 16: 
61ff.). Auf Nachfrage sind zum Frühjahr 2020 ca. 150 Mitglieder in der BAG organisiert, wobei der Mitgliederstatus von freiberuflichen Wohnberater*innen bis hin zu ganzen Kommunen sehr unterschiedlich definiert wird (IP 1: 88). Hier zeigt sich, dass die Bundesarbeitsgemeinschaft zwar einerseits durchaus als Dachverband der Wohnberatung wahrgenommen wird, aber andererseits nur über eine begrenzte Organisationsmacht verfügt.

\subsubsection{Die Wohnberatungen auf Ebene der Bundesländer}

Im Folgenden stehen die Wohnberatungen in den 16 Bundesländern im Fokus: Welche Strukturen zu Trägerschaften und der regionalen Verbreitung der Wohnberatungsstellen sind zu identifizieren? Um zu überprüfen, inwieweit die Wohnberatung als tatsächliche politische Maßnahmen gelten kann, wird sich danach der Finanzierung dieser Beratungsangebote und insbesondere der Einrichtung von landesweiten Fachstellen gewidmet.

Struktur der Wohnberatungsstellen

Wie bereits skizziert, entstanden die ersten Wohnberatungsstellen in den 1980er Jahren. Die Initiator*innen waren zumeist einzelne Kommunen oder Wohlfahrtsverbände (BAG Wohnungsanpassung 2019; Lehrmacher-Dubberke et al. 2015: 143). Aus diesen vereinzelten Anstrengungen entwickelte sich die heutige heterogene Struktur an Wohnberatungsstellen. Wohnberatungen werden von kommunalen Sozial- oder Wohnungsämtern, Pflegestützpunkten und Seniorenberatungen, kirchlichen und freien Wohlfahrtsverbänden, von Architekten- und Handwerkskammern oder Ergotherapeut"innen sowie Krankenkassen und Pflegediensten angeboten. Dieser Kreis an Akteuren ist nicht abschließend zu verstehen, vielmehr entwickelt sich das Feld dynamisch fort und es haben mittlerweile auch Wohnungsunternehmen selbst entsprechende Wohnberater*innen in ihrem Mitarbeiterstamm, zudem finden sich freiberufliche Wohnberater*innen ohne institutionelle Anbindung (BMFSFJ 2009: 16; BMVBS 2013: 41; BMUB 2016: 27; BMFSFJ 2018: 22f.; Lehrmacher-Dubberke et al. 2015: 144; Schneider 2013: 157). Unabhängig von der Trägerstruktur werden Wohnberatungen vielfach lediglich mit einem gewissen Stellenanteil neben einer weiteren Tätigkeit ausgeführt, etwa neben der hauptsächlichen Arbeit eines Pflegestützpunktes (IP 54: 41ff.; BMVBS 2011: 74). Die Wohnberater*innen haben wiederum ganz unterschiedliche fachliche Hintergründe - von Sozialarbeiter*innen über Architekt*innen und Techniker*innen bis hin zu unterschiedlichen Pflegefachberufen (Wohnberatungsstelle-1: 10; BMFSFJ 2009: 17).

Verschiedene Studien haben in den letzten Jahren versucht, diese Heterogenität einzufangen und zu erfassen, wie viele entsprechende Beratungsstellen es überhaupt gibt. Die Erkenntnisse variieren von bundesweit ca. 350 Beratungsangeboten (BMVBS 2011: 74; Lehrmacher-Dubberke et al. 2015: 143) bis hin zu deutlich höheren Schätzungen - je nachdem, welche Beratungsleistungen mitgedacht werden. Unterschieden nach örtlichen Beratungsstellen ( $\mathrm{N}$ 362) und Pflegestützpunkten ( $\mathrm{N}$ 401) kommt die Studie von KDA/Wüstenrot Stiftung (2014a: 158ff.) zu höheren Angaben, jedoch noch ohne dabei die Beratungsangebote von freien Trägern oder Handwerker- und Architektenkammern zu berücksichtigen. In Bayern und Nordrhein-Westfalen gibt 
es äquivalent zur Bundesarbeitsgemeinschaft zudem Landesarbeitsgemeinschaften (LAG) zur Wohnungsanpassung (Lehrmacher-Dubberke et al. 2015: 144). Aber auch eine Übersicht der LAG Bayern zu den Beratungsstellen im Bundesland verweist lediglich auf »eine Liste der uns bekannten Wohnberatungsstellen (LAG Bayern 2019: 2).

Durch die ungeschützte Berufsbezeichnung und die vielfältigen Akteure ist das Feld der Wohnberatung also äußerst unübersichtlich. Dennoch sind regionale Unterschiede festzustellen, was die Verteilung der Beratungsangebote betrifft: Während NordrheinWestfalen als einziges Bundesland ein flächendeckendes Netz an einschlägigen Wohnberatungen vorzuweisen hat, ist die Beratung in Berlin, im Saarland und in Niedersachsen eher an Pflege- oder Seniorenstützpunkte angedockt (Wohnberatungsstelle-3: 23f.; Wohnberatungsstelle-5: 3f.; Wohnberatungsstelle-6: 15f.). Berlin hatte seit $1992 \mathrm{zu}$ nächst pro Stadtbezirk eine Koordinierungsstelle »Rund ums Alter« institutionalisiert, welche seit 2009 in einer Doppelstruktur zu den daraufhin eingerichteten Pflegestützpunkten bestehen (Wohnberatungsstelle-2: 14ff.).

Mit der Trägerstruktur und der eher flächendeckenden oder eher punktuellen Verbreitung von Wohnberatungsstellen verbunden sind auch Ressourcen zu weiteren, über die reine Beratung hinausgehenden Aktivitäten. Generell arbeiten Wohnberater*innen mit zahlreichen weiteren Akteuren der Bereiche "Wohnen« oder "Gesundheit« zusammen: Von Krankenhäusern und Ärzt*innen über Ergotherapeut*innen, aber auch mit den örtlichen Wohnungsunternehmen (Wohnberatungsstelle-1: 28; Wohnberatungsstelle-4: 6). So wird eine Beratungsstelle bei neuen Bauprojekten explizit mit ihrer Expertise einbezogen (Wohnberatungsstelle-6: 23). Andernorts gab es bis vor einigen Jahren einen »Runden Tisch im Alter«, wo auch mit der lokalen Wohnungswirtschaft konstruktiv zusammengearbeitet wurde. Durch die inzwischen angespannte Lage auf den Wohnungsmärkten bestünde derzeit jedoch weniger Interesse vonseiten der Wohnungsunternehmen, sich an Maßnahmen zur Wohnungsanpassung zu beteiligen (Wohnberatungsstelle-2: 23).

Die Expert*inneninterviews zeigen schließlich, dass auch die regionalen Ansprechpartner*innen der BAG vielfach keinen Überblick über die uneinheitliche Struktur der Wohnberatungen in ihrem Bundesland haben: "Ich weiß nicht wirklich, ob ich Ihnen viele Informationen liefern kann. Leider ist in BUNDESLAND XY [Anonymisierung durch die Autorin] eine Vernetzung nicht gegeben und auch das Thema Wohnberatung ist eher dünn" (Wohnberatungsstelle-5: 1). Andernorts recherchieren engagierte Wohnberater*innen jährlich aufs Neue, um zu prüfen, ob neue Anbieter*innen von Wohnberatungen hinzugekommen sind (Wohnberatungsstelle-6: 9f.). Aufgrund dieser undurchsichtigen Datenlage sind die bisherigen Ausführungen als tendenziell zu werten.

Finanzierung der Wohnberatungsstellen

Die bisherigen Ausführungen zur Struktur der Wohnberatungsstellen sind maßgeblich abhängig von deren Finanzierung. Es soll hier nicht um die Finanzierung der Wohnungsanpassung selbst gehen - dies wäre ein Bestandteil des ökonomischen Instru- 
mentariums -, sondern um die Finanzierung der Wohnberatung als Beratungsleistung. ${ }^{10}$

Mit der diversen Struktur der Wohnberatung selbst hängen unterschiedlichste Formen der Finanzierung zusammen (Wohnberatungsstelle-1: 7; BMVBS 2011: 75). In diesem Kontext argumentiert Joo (2018: 117ff.), dass sich Kommunen und Pflegekassen an der Finanzierung beteiligen sollten. Sie gelten ihr als Profiteure der Wohnungsanpassung, da somit Heimaufenthalte verzögert würden, die wiederum mit entsprechenden Mehrkosten verbunden wären. In der empirischen Überprüfung stellt die Autorin jedoch eher selten ein finanzielles Engagement der Pflegekassen an den Beratungskosten fest (ebenda: 119). In Baden-Württemberg findet teilweise eine fallbezogene Kostenerstattung statt. Lediglich in Nordrhein-Westfalen beteiligen sich die Pflegekassen durch einen pauschalen Anteil, wobei das Land bzw. die jeweilige Kommune einen 50prozentigen Anteil mitfinanziert. Diese Mischfinanzierung ist auf eine Initiative des Landes Nordrhein-Westfalen in den 1990er Jahren zurückzuführen und erklärt das flächendeckende Netz an Wohnberatungen in diesem Bundesland (vgl. KDA/Wüstenrot Stiftung 2014a: 68; Lehrmacher-Dubberke et al. 2015: 143).

Abgesehen vom »Sonderfall« Nordrhein-Westfalen ist die Finanzierung in den anderen Bundesländern selten institutionalisiert. Während eine Förderung von Modellprogrammen nicht unüblich ist, bleibt eine verstetigte Finanzierung der Wohnberatung vielfach aus (KDA/Wüstenrot Stiftung 2014a: 68; Lehrmacher-Dubberke et al. 2015: 143). Die Folge ist ein Ungleichgewicht zwischen Haupt- und Ehrenamtlichen zugunsten letzterer, wobei die ehrenamtlichen Wohnberater*innen beispielsweise durch eine Mitarbeiterin in einem Pflegedienst koordiniert werden (Wohnberatungsstelle-1: 11f.). Eine interessante Ausnahme bildet Bayern. Hier unterstützt das Bayerische Staatsministeriums für Arbeit und Soziales, Familie und Integration den Aufbau von Wohnberatungsstellen mit einer Anschubfinanzierung von bis zu 40.000 Euro. Mit dieser Förderung sind einige Voraussetzungen verbunden, so ist zur Vermeidung von isolierten zusätzlichen Strukturen eine Anbindung an bestehende Beratungsangebote anzustreben und es wird eine Zusammenarbeit mit der Beratungsstelle Barrierefreiheit der Bayerischen Architektenkammer eingefordert (Koordinationsstelle Wohnen im Alter 2020). Insgesamt loben verschiedene Wohnberater*innen aus anderen Bundesländern dieses Modell der integrierten Fördervoraussetzungen zur stärkeren Qualitätssicherung (IP 1: 28ff.; IP 54: 39).

Neben dem flächendeckenden Angebot der Wohnberatungsstellen in NordrheinWestfalen, finanziert anteilig von den Pflegekassen und dem Land bzw. den Kommunen, sowie der Anschubfinanzierung in Bayern, ist im Folgenden einem dritten Aspekt der Finanzierung gesonderte Aufmerksamkeit zuzukommen: der Einrichtung landesweiter Koordinierungs- oder Fachstellen.

10 Die Wohnungsanpassung selbst wird am häufigsten über den Zuschuss für wohnumfeldverbessernde Maßnahmen der Pflegekassen oder aus Eigenmitteln finanziert, seltener über KfW- oder Landesförderprogramme (Wohnberatungsstelle-1: 24; Wohnberatungsstelle-5: 13; Wohnberatungsstelle-6: 28ff.) 
Landesweite Fachstellen zur Wohnberatung

Von verschiedenen Gesprächspartner*innen wird eine landesweite Fachstelle zur Koordination, zum Wissensaustausch und teils auch zur Qualifizierung und Fortbildung als sehr wichtig eingestuft. Ferner würde durch eine zuständige und anerkannte Fachstelle die Anbindung an die Landespolitik erleichtert, was zu einer besseren Interessenvertretung der Wohnberatungen im Land selbst beitragen könne (Wohnberatungsstelle-3: 11ff., 25; IP 1: 32ff.; vgl. auch BMFSFJ 2006: 75). Die Rechercheergebnisse weisen solche koordinierenden Strukturen für Bayern, Hessen, Niedersachsen, Nordrhein-Westfalen und Rheinland-Pfalz nach.

Die Idee der Fachstellen ist dabei jünger als die der eigentlichen Wohnberatungsstellen: Ende der 1990er Jahre unterstützt das Modellprogramm »Selbstbestimmt Wohnen im Alter« des damaligen Bundesministeriums für Familie, Senioren, Frauen und Jugend die Einrichtung entsprechender Stellen in Niedersachsen, Hessen und Bayern (Schneider 2013: 156). Die Idee zu diesem Modellprogramm wiederum entstand im Rahmen des Zweiten Berichts der Bundesregierung zur Lage der älteren Generationen, welcher dem Schwerpunktthema »Wohnen im Alter« gewidmet war (BMFSFJ 1999: 1). Hier wurde erkannt, dass auf lokaler Ebene bereits unterschiedlichste Beratungsstrukturen bestünden, diese jedoch in ihrer Verbreitung und Qualität sehr unterschiedlich seien: »Die Schaffung der institutionellen Rahmenbedingungen hatte großen Einfluß aufdie bisherige Entwicklung«, lag damals doch die Hälfte der bestehenden Beratungsstellen allein in Nordrhein-Westfalen und Baden-Württemberg (Deutscher Bundestag 1998: 111). In beiden Bundesländern gab es seit Beginn der 1990er Jahren eine landesweite Koordination, welche das Bundesmodellprogramm als Erfolgsgarant zur Ausbreitung und Qualitätssicherung der Wohnberatung betrachtete (BMFSFJ 1999: 1). Zusätzlichen Aufwind bekamen die landesweiten Fachstellen durch die Einführung der Pflegeversicherung im Jahr 1995, weil durch den damit verbundenen Zuschuss für wohnumfeldverbessernde Maßnahmen eine stärker gesicherte bauliche Qualifikation in der Beratung eingefordert wurde (Deutscher Bundestag 1998: 111). Diese Entwicklungen führten dazu, dass die Bundes- und teils auch Landespolitik den Bedarf einer politischen Steuerung in der Wohnberatung anerkannte.

Dieser chronologische Abriss ist auch deshalb wichtig, da er die frühe Entwicklung der Wohnberatung und der landesweiten Koordination nachzeichnet. Als pfadabhängiges System nutzen die damals in den Modellprojekten berücksichtigten Länder ihre Erfahrungen und Institutionalisierungen noch heute (IP 1: 32ff.). Gleichzeitig wird das Konzept einer »Fachstelle« durchaus unterschiedlich interpretiert: Während die Hessische Fachstelle für Wohnberatung sich stärker auf die Koordination und Qualifizierung der Wohnberatungsstellen selbst konzentriert, erscheinen das NIEDERSACHSENBÜRO Neues Wohnen im Alter sowie die Koordination Wohnberatung NRW stärker in landespolitische Prozesse eingebunden zu sein. Andersherum wurde in Rheinland-Pfalz keine neue Struktur geschaffen, sondern die dortige Landesberatungsstelle Barrierefrei Bauen dockt sich bei der Verbraucherzentrale des Landes in Kooperation mit der Architektenkammer an. Die Koordinationsstelle Wohnen im Alter in Bayern hingegen ist an eine bestehende Forschungs- und Beratungsstelle angeschlossen worden. 
Gemein ist diesen Fachstellen, dass sie über die jeweiligen Sozialministerien der Bundesländer gefördert werden. Dabei variiert das Ausmaß dieser Landesförderung wiederum von Land zu Land. Für Hessen ließ sich für das Jahr 2015 eine Förderung von 65.000 Euro feststellen (Hessischer Landtag 2015b: 1), wobei in der Zwischenzeit eine Stellenaufstockung auf insgesamt 1,75 Vollzeitstellen stattgefunden hat - und damit mutmaßlich eine Steigerung der Fördersumme (IP 16: 8ff.). Niedersachsen finanziert aktuell zwei $50 \%$-Stellen und in Rheinland-Pfalz werden die Wohnberater*innen in den Verbraucherzentralen zu $100 \%$ aus dem Sozialministerium gefördert (Wohnberatungsstelle-3: 3ff., 22f.; Wohnberatungsstelle-4: If.). Bremen verfügt zwar über keine eigene koordinierende Fachstelle, unterstützt aber den gemeinnützigen Verein Wohnberatung komfort e.V. als »zentrale Anlaufstelle im Land« mit $89.000 \mathrm{Eu}$ ro jährlich (Bremische Bürgerschaft 2015: 4f.). In Nordrhein-Westfalen teilen sich das Ministerium für Arbeit, Gesundheit und Soziales sowie die Landesverbände der Pflegekassen und der Verband der Privaten Krankenversicherung die Finanzierung der hauptamtlichen Wohnberater*innen (Koordination Wohnberatung NRW 2020). Außerhalb von Nordrhein-Westfalen sind die Fachstellen häufig auf zeitlich befristete Förderungen angewiesen, womit eine jährliche Beantragung der neuerlichen Finanzierung und damit eine stete Unsicherheit der Arbeit selbst verbunden ist (IP 16: 8ff.; Koordination Wohnberatung NRW 2020; Wohnberatungsstelle-3: 3ff., 22f.). Das zeigt sich in einem Bundesland, in dem für gut zehn Jahre eine zentrale Fachstelle zur Wohnungsanpassung bestand und vom Land entsprechend finanziert wurde, bis "das Ganze durch fragmentierte politische Verantwortlichkeiten eingeschlafen ist (Gedächtniszitat nach Wohnberatungsstelle-1: 7f).

Mit den bisherigen Fachstellen wurden solche fokussiert, die einschlägig auf die Wohnungsanpassung zugeschnitten sind. Die Studie von KDA/Wüstenrot Stiftung (2014a: 158ff.) wählt einen breiteren Ansatz und integriert Koordinierungsstellen zu gemeinschaftlichen Wohnformen (Berlin, Niedersachsen, Schleswig-Holstein) oder Pflegewohngemeinschaften (Brandenburg, Hamburg). Die vorliegende Arbeit wählt stattdessen eine enge Arbeitsdefinition, nach der landesweite Fachstellen zur Wohnberatung dezidiert den Schwerpunkt der Wohnungsanpassung verfolgen müssen. Dadurch wird dem Erkenntnisinteresse zur baulichen Barrierefreiheit Rechnung getragen, womit ein wohnungspolitischer Fokus einhergeht und sozialpolitische - vor allem pflegepolitische - Aspekte ausgeklammert sind (vgl. Kapitel 1).

Insgesamt bleibt festzuhalten: Fachstellen zur Wohnungsanpassung sind eine Möglichkeit, die von den Bundesländern vergleichsweise häufig genutzt wird, um die ansonsten weitestgehend selbstgeregelte Wohnberatung politisch zu unterstützen. Gleichzeitig sei darauf hingewiesen, dass die bloße Existenz einer landesweiten Fachstelle noch kein Garant für entsprechende Qualitätsstandards und Vernetzungen darstellt. Umgekehrt müssen Beratungsangebote in Ländern ohne Fachstelle keinesfalls automatisch eine geringere Beratungsqualität aufweisen. 


\subsubsection{Fazit zu Wohnberatungsstellen}

In der Zusammenschau gelten Bayern und Nordrhein-Westfalen als prominente Bundesländer in der politischen Steuerung der Wohnberatung (IP 54: 39ff.; IP 1: 28). Neben dem besonderen Finanzierungsmodell in Nordrhein-Westfalen und der bayerischen Anschubfinanzierung sind diese beiden Länder die einzigen mit etablierten Landesarbeitsgemeinschaften zur Wohnungsanpassung. Gleichzeitig beschrieb eine Gesprächspartnerin die Wohnberatung in Deutschland im Sinne von zwei Lagern, wobei sie Nordrhein-Westfalen eine eher träge, pfadabhängige Logik des »Es war schon immer so« zuschreibt, wohingegen Bayern innovativer auf neue Herausforderungen reagieren würde (Wohnberatungsstelle-2: 6ff.). Dieser Eindruck wird dadurch verstärkt, dass die bestehende Sekundärliteratur zwar häufig auf das flächendeckende Netz der Wohnberatungen im Nordwesten verweist, die Interviewpartner*innen jedoch wiederholt das flexiblere bayerische Modell betonen (Wohnberatungsstelle-1: 25; Wohnberatungsstelle-5: 17). Mutmaßlich spielt hier eine Rolle, dass der nordrheinwestfälische Schulterschluss mit den Pflegekassen nur begrenzt auf andere Länder übertragbar scheint - nicht zuvorderst Widerstände aus den Pflegekassen, sondern die kommunale Gegenfinanzierung könnte hier ein Hindernis darstellen (IP 1: 79ff.).

Neben Bayern und Nordrhein-Westfalen steuern aber auch Hessen, Niedersachsen und Rheinland-Pfalz die Wohnberatung in ihrem Land durch die dort angesiedelten Fachstellen. In Rheinland-Pfalz ist zudem die enge Anbindung an die Architektenkammer hervorzuheben. Diese reagiert - ähnlich wie die Fördervoraussetzungen in der bayerischen Anschubfinanzierung - auf die rechtlich ungeklärte Berufsbezeichnung »Wohnberater*in« und gewährleistet eine Qualitätssicherung über speziell weitergebildete Architekt*innen (Wohnberatungsstelle-4: 2). Darüber hinaus wurden in den Gesprächen immer wieder einzelne Kommunen hervorgehoben, die im Rahmen ihrer kommunalen Steuerungsmöglichkeiten eine entsprechende Beratungsinfrastruktur aufbauen (Wohnberatungsstelle-2: 2; IP 1: 28ff.).

Insgesamt zeigen die Entwicklungen der Wohnberatungen in den 1980er und 1990er Jahren eine starke Pfadabhängigkeit. Niedersachsen, Hessen und Bayern haben im Bundesmodellprogramm "Selbstbestimmt Wohnen im Alter" entsprechende Fachstellen eingerichtet. Auch wenn diese nicht überall verstetigt worden sind, wirken diese Aktivitäten bis heute nach und bestehen - teils in modifizierter Form - fort. Durch die landesweite Koordination schon Anfang der 1990er Jahre galten NordrheinWestfalen und Baden-Württemberg damals als Vorreiter, haben aber in der Zwischenzeit unterschiedliche Wege eingeschlagen: Während sich in Nordrhein-Westfalen die flächendeckende Versorgung mit Wohnberatungsstellen herausgebildet hat, konnte die Fachstelle im Südwesten nicht aufrechterhalten werden (Wohnberatungsstelle-1: 7f). Insgesamt ist die Landesfinanzierung der Fachstellen vielfach von mittelbaren Förderungen abhängig, womit diese trotz der skizzierten Pfadabhängigkeiten nur bedingt als fest etablierte Struktur gelten können. Im Falle einer veränderten politischen Prioritätensetzung im Land sind die Hürden für inhaltliche oder budgetäre Kürzungen bis hin zu Schließungen also geringer als bei einer festen Institutionalisierung.

Abschließend handelt es sich bei den Wohnberatungsstellen in einigen Ländern um eine informationelle politische Maßnahme, in anderen Ländern stärker um eine 
Selbstregelung über freie Träger, Wohnungsunternehmen oder selbstständige Wohnberater*innen (vgl. Kapitel 4.2.1). Vielfach sind es zudem Kommunen, die im Rahmen ihres kommunalen Aufgabenspektrums Wohnberatungen anbieten und dabei - teilweise - durch landespolitische koordinierende Strukturen unterstützt werden. Joo (2018) hat diese Gemengelage unter dem Schlagwort »Wohlfahrtskorporatismus« untersucht. In ihrer Lesart findet eine "Sozialpolitisierung der vormals privaten Räume« statt, wobei der Markt der Wohnberatung aktuell durch ein »komplexes Verhältnis zwischen marktwirtschaftlichen und sozialpolitischen Elementen gekennzeichnet « ist (Joo 2018: 162ff.). Zwar ist mit der Sozialpolitisierung eine zunehmende politische Steuerung verbunden, jedoch kann damit noch keine Aussage darüber getroffen werden, wie qualitativ hochwertig die tatsächlichen Beratungen letztlich sind. Je nach Struktur vor Ort seien gerade die kurzen Wege wichtig und mehrere Wohnberater*innen bewerten diese von außen verworrene Struktur unterschiedlichster Beratungsakteure nicht unbedingt als negativ. So könne z.B. eine Integration in bereits bestehende Beratungsangebote sinnvoller sein als der Aufbau einer Parallelstruktur (Wohnberatungsstelle-3: 25). Aus politikfeldanalytischer Perspektive erschweren diese politikfeldübergreifenden Beratungsangebote die Analyse allerdings. Insbesondere die Pflegekassen sind ein Akteur, der aus wohnungspolitischer Sicht bislang noch nicht aufgeschienen war. Hier zeigt sich klar der Querschnittscharakter des barrierefreien bzw. -reduzierten Wohnraums, der einerseits in wohnungspolitischen und andererseits in sozialpolitischen Handlungsfeldern zu verorten ist.

\subsection{Fazit}

Kapitel 6 hat untersucht, in welchen Ausprägungen die Bundesländer politische Maßnahmen zur Steuerung von barrierefreiem bzw. -reduziertem Wohnraum nutzen. Diese Erkenntnisse werden im Folgenden zusammengefasst. Zur Übersicht dient zunächst der zugrundeliegende Policy-Mix, der auf Basis der gewonnenen Erkenntnisse eine inhaltliche Verfeinerung erfährt. Daraufhin sind die einzelnen Policy-Ausprägungen von Interesse: Welche Bundesländer konnten als besonders aktiv in der politischen Steuerung identifiziert werden? Diese Analyseergebnisse sind anschließend zu ergänzen und abzugleichen mit den Einschätzungen aus dem Feld - von den Interviewpartner*innen wurde dafür die Nennung sogenannter Vorreiter-Länder erbeten.

Policy-Mix zum barrierefreien bzw. -reduzierten Wohnraum

Tabelle 9 zeigt den untersuchten Policy-Mix zum regulativen Bauordnungsrecht, den Förderprogrammen der Landesförderbanken und den Wohnberatungsstellen. Dabei sind zwei Unterscheidungslinien wichtig, die im Rahmen der Analyse immer wieder deutlich geworden sind. 